\title{
地震作用下岩体边坡针固界面剪切作用数值模拟 分析
}

龙哲 ${ }^{1}$, 言志信 ${ }^{1,2 *}$, 周小亮 ${ }^{1}$

1. 兰州大学土木工程与力学学院, 兰州 730000 ;

2. 河南城建学院土木与交通工程学院, 平顶山 467000

*E-mail: yzx10@163.com

收稿日期: 2017-06-08; 接受日期: 2017-08-14; 网络出版日期: 2017-09-14

摘要为研究地震作用下针固岩体边坡中针杆-砂浆界面和砂浆-岩体界面的剪切相互作用, 揭示针固界面上 相互作用机理, 同时获得地震作用下针固岩体边坡两针固界面的剪应力分布规律, 采用有限差分软件FLAC $\mathrm{FL}^{3 \mathrm{D}}$ 建立针固岩体边坡数值分析模型, 并用interface接触面单元模拟针杆-砂浆界面和砂浆-岩体界面的剪切相互作 用, 对简谐地震波作用下针固边坡中全长黏结针杆针固界面剪应力的分布规律进行了研究, 研究发现: 在地震 作用下, 砂浆-岩体界面剪应力首先达到其极限黏结强度并出现脱黏现象, 剪应力峰值从破坏面向锚杆两端发 展; 与此同时, 针杆-砂浆界面上的剪应力没有达到其极限黏结强度, 但是该界面剪应力峰值会随着砂浆-岩体 界面的脱黏而进行相应的调整; 砂浆一岩体界面脱黏后, 针杆轴力呈两翼外凸的人字形曲线分布, 轴力曲线拐点 与界面剪应力曲线峰值点相对应, 脱秥区域针固体均匀拉伸, 轴力分布均匀. 该研究可为相关模型试验、现场 监测、设计施工研究提供参考.

关键词针固边坡, 接触面单元, 界面剪应力, 针杆-砂浆界面, 砂浆-岩体界面

\section{1 引言}

岩土体针固技术因其具有结构巧、施工易、造 价低、效果好等特点, 近年来在国内外得到了广泛的 应用. 在地震作用下, 针固边坡中针固界面上的剪切 作用加剧, 可能导致锚杆从砂浆中拔出、针杆连同砂 浆从针孔中拔出等典型针固破坏现象, 即锚杆-砂浆界 面或砂浆-岩体界面出现剪切破坏, 进而造成各种工程 事故. 开展对地震作用下岩体边坡针固界面上的剪切 相互作用问题的深入系统研究, 揭示针固界面上的剪
切相互作用及其与边坡地震响应的关系, 是进行针固 边坡动力稳定性分析和地震失稳机理研究的关键, 具 有重要的理论意义和工程应用价值.

目前, 静力拉拔荷载作用下的针杆界面剪应力问 题已经比较成熟, 诸多研究人员对这个问题开展了深 入研究 ${ }^{[15]}$. 而对动力作用下锚杆界面剪应力的研究 较少, 此前在动力作用下针杆针固方面的研究主要有: 叶海林等人 ${ }^{[6]}$ 和Zhou等人 ${ }^{[7]}$ 利用有限差分软件FLAC ${ }^{3 \mathrm{D}}$ 研究了动荷载作用下针固边坡稳定性问题; Mortazavi 和Tabatabaei Alavi ${ }^{[8]}$ 利用FLAC ${ }^{3 \mathrm{D}}$ 分析了动荷载作用下,

引用格式: 龙哲, 言志信, 周小亮. 地震作用下岩体边坡针固界面剪切作用数值模拟分析. 中国科学: 技术科学, 2017, 47: 1049-1056

Long Z, Yan Z X, Zhou X L. A numeral simulation of anchored interface shear stress for bolt in rock mass slope under earthquake (in Chinese). Sci Sin Tech, 2017, 47: 1049-1056, doi: 10.1360/N092017-00171 
全长黏结锚杆支护地下空间时针杆的轴力和位移分 布规律; 言志信等人 ${ }^{[9]}$ 利用有限差分法和MATLAB语 言, 分析了针固系统的动力响应问题; 刘建华等人 ${ }^{[10]}$ 根据Pseudo-static分析原理, 研究了简谐荷载下针固边 坡中的针杆受力问题.

由上述可知, 迄今为止动力作用下针杆针固界面 剪应力这个方面已经有了一定的研究基础, 但是前人 在研究界面剪应力时往往将针杆-砂浆之间和砂浆-岩 体之间形成的两个界面简化成一个针固界面来研究针 杆针固界面剪应力分布规律 ${ }^{[11,12]}$. 而参考前人研究 ${ }^{[13]}$ 和现行规范 ${ }^{[14]}$ 可知, 全长黏结针杆水泥砂浆与钢筋间 的黏结强度设计值的范围在2.1 2.7 MPa之间, 说明使 用不同强度砂浆对针杆-砂浆界面的黏结强度影响不 大. 但砂浆-岩体界面随着岩性单轴抗压强度从低到高, 岩体与针固体的极限黏结强度标准值也从 $0.27 \mathrm{MPa}$ 增 长到2.6 MPa, 相差10倍左右. 根据岩体性能的不同, 锚 杆-砂浆界面和砂浆-岩体界面的破坏方式也不同, 所 以同时得到两个界面上的剪应力, 对于揭示针固界面 上的剪切相互作用及其与边坡地震响应的关系是很 有必要的. 而目前针对动力作用下两个界面的剪切相 互作用进行的试验研究存在着测试难度大、测试结 果不理想 ${ }^{[15,16]}$ 等问题, 因此需要通过数值模拟研究进 行进一步的探索, 为将来的试验研究提供指导.

\section{2 建模方式的选取}

目前岩土工程方面常用的数值模拟软件大多拥 有能够模拟针杆与岩土体相互作用的特殊单元, 其中 如ANSYS中的link单元、FLAC ${ }^{3 D}$ 中的cable单元等, 可 以模拟针杆(索)支护岩土体的效果. 但此类单元是实 际结构的一种“抽象”简化, 难以真实地反映针杆与岩 土体的相互作用. 这类简化单元往往只能通过软件预 设的方法得到简化单元内轴力、剪应力的轴向平均 值, 无法提取出错杆-砂浆界面和砂浆-岩体界面这两 个界面上沿针杆轴向、周向的剪应力分布规律.

在经过大量尝试后, 最终选用实体单元模拟针 杆、砂浆、岩体三部分, 用 $F L A C^{3 \mathrm{D}}$ 自带的interface接 触面单元模拟针杆、砂浆、岩体三者之间的两个界面. 接触面和实体单元黏结后, 由法向、切向刚度及滑动 的特性来影响接触面的特征. 结合式(1)和(2)及图1可 知, 首先计算目标面和接触面节点的法向和切向速度,

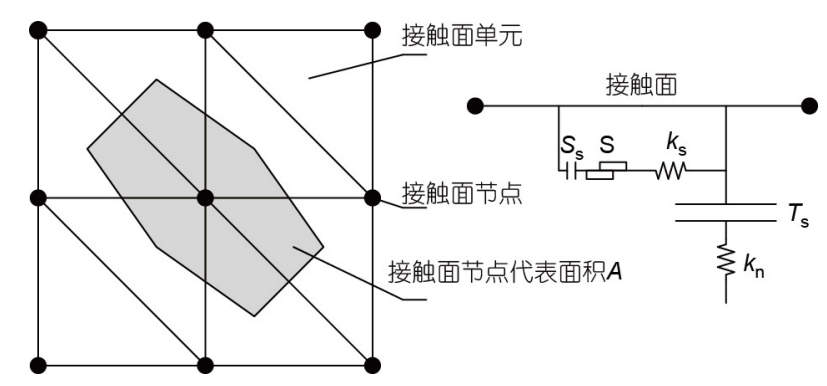

图 1 接触面单元力学原理示意图

接着计算其对应的法向力和切向力, 其计算公式如下:

$$
\begin{aligned}
& \boldsymbol{F}_{n}^{(t+\Delta)}=\boldsymbol{k}_{n} u_{n} A+\boldsymbol{\sigma}_{n} A, \\
& \boldsymbol{F}_{s i}^{(t+\Delta)}=\boldsymbol{F}_{s i}^{(t)}+\boldsymbol{k}_{s} \Delta \boldsymbol{u}_{s i}^{(t+0.5 \Delta)} A+\boldsymbol{\sigma}_{s i} A,
\end{aligned}
$$

式中, $F_{n}^{(t+\Delta t)}$ 为时刻 $t+\Delta t$ 的法向力矢量; $\boldsymbol{F}_{s i}^{(t+\Delta t)}$ 为 $t+\Delta t$ 时 刻的切向力矢量; $u_{n}$ 为接触面和目标面之间的绝对位 移; $\Delta \boldsymbol{u}_{s i}$ 为相对剪切位移增量矢量; $\boldsymbol{\sigma}_{n}$ 为法向应力; $\boldsymbol{\sigma}_{s i}$ 为 切向应力; $\boldsymbol{k}_{n}$ 为法向刚度; $\boldsymbol{k}_{s}$ 为切向刚度; $A$ 为接触面 节点代表面积.

当接触面上的切向应力大于抗剪强度 $S_{s}$ 时, 接触 面由弹性进入塑性阶段, 滑块 $\mathrm{S}$ 发生脱黏滑动. 当接触 面上的法向应力大于抗拉强度 $T_{s}$ 时, 接触面发生破坏.

采用实体单元结合接触面单元的方法来建立针 固边坡模型, 能直接提取接触面单元上的剪应力作为 界面剪应力, 最为精确合理. 且通过该方法还可以得 到针杆、砂浆内部及针杆-砂浆界面、砂浆-岩体界面 上沿针杆轴向、周向的剪力分布规律. 从而达到从多 方面入手, 深入分析地震作用下岩体边坡针固界面上 的剪切相互作用的目的.

\section{3 针固边坡的数值模拟}

\section{1 边坡模型的建立}

一针固岩体边坡模型如图2所示, 坡高 $12 \mathrm{~m}$, 坡角 $90^{\circ}$, 为垂直边坡, 边坡宽度 $3 \mathrm{~m}$, 岩体密度 $2500 \mathrm{~kg} / \mathrm{m}^{3}$; 边坡中存在一个垂直的软弱结构面, 结构面厚度 $0.5 \mathrm{~m}$; 边坡岩体和软弱结构面的物理力学参数见表 1 .

\section{2 针杆及界面参数的选取}

边坡采用全长黏结针杆进行支护, 其中针孔直径 $D=120 \mathrm{~mm}$, 针固注浆材料采用 $\mathrm{M} 30$ 水泥砂浆, 针杆直 


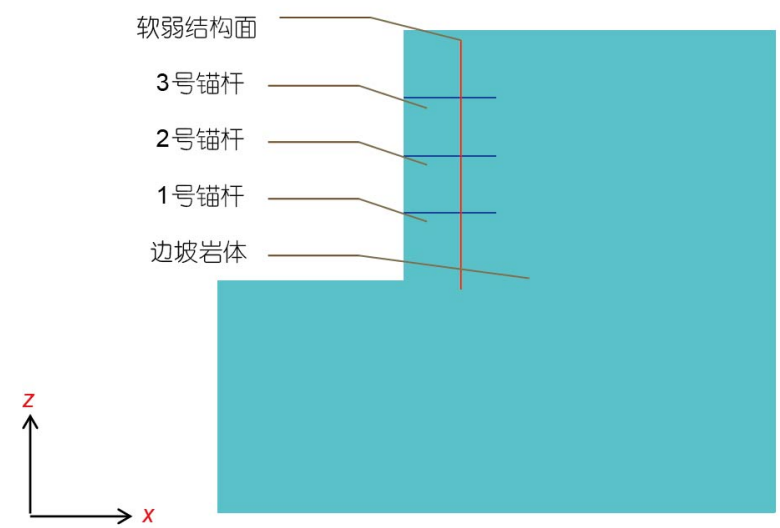

图 2 (网络版彩图)针固边坡数值模型

表 1 边坡材料参数

\begin{tabular}{cccccc}
\hline 类别 & $\begin{array}{c}\text { 弹性模 } \\
\text { 量 }(\mathrm{GPa})\end{array}$ & 泊松比 & $\begin{array}{c}\text { 黏聚力 } \\
(\mathrm{MPa})\end{array}$ & $\begin{array}{c}\text { 内摩擦 } \\
\text { 角 }\left({ }^{\circ}\right)\end{array}$ & $\begin{array}{c}\text { 抗拉强 } \\
\text { 度 }(\mathrm{MPa})\end{array}$ \\
\hline $\begin{array}{c}\text { 边坡 } \\
\text { 岩体 }\end{array}$ & 1 & 0.25 & 1 & 40 & 0.6 \\
$\begin{array}{c}\text { 软弱结 } \\
\text { 构面 }\end{array}$ & 0.5 & 0.25 & 0.1 & 30 & 0.06 \\
\hline
\end{tabular}

径 $R=40 \mathrm{~mm}$, 弹性模量 $E=210 \mathrm{GPa}$, 密度为 $7800 \mathrm{~kg} / \mathrm{m}^{3}$, 针杆垂直于坡面布设, 倾角为 $0^{\circ}$, 间距为 $3 \mathrm{~m}$, 长度 $L=8 \mathrm{~m}$. 针杆与软弱结构面呈 $90^{\circ}$ 夹角, 软弱结构面 处于针杆中部, 即距离针头3.5 4 $\mathrm{m}$ 的区域.

针固体建模见图3, 针杆-砂浆、砂浆-岩体这两个 界面上的极限黏结强度是计算界面剪应力的关键参 数, 当界面剪应力超过该值时, 界面开始出现脱黏现 象. 参考《建筑边坡工程技术规范GB50330-2013》中 关于针杆设计计算时的强度参数, 针杆-砂浆界面的极 限黏结强度取 $2.4 \mathrm{MPa}$, 砂浆-岩体界面的极限黏结强 度取 $0.6 \mathrm{MPa}$.

\section{3 边界条件设置}

在动力模拟时, 模型边界上存在波的反射, 对动力 分析的结果造成影响. 由于本研究中的关键构件之一 为针杆, 其相对于整个针固边坡很小, 所以对整个边 坡模型的边界条件设置要求很高, 只有正确地处理好 边界问题, 才能减小模型边界造成的误差, 保证动力 分析的结果能真实反映实际的针固界面的反应过程.

$F{ }^{3} C^{3 D}$ 动力分析模块提供了静态边界和自由场

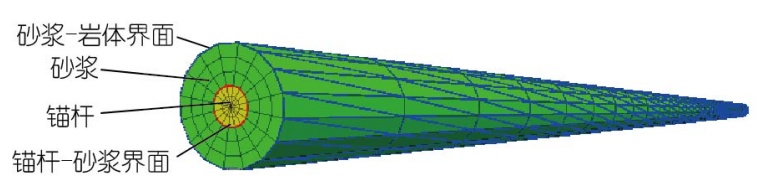

图 3 (网络版彩图)针固体数值模型

边界, 由于研究的是岩体边坡问题, 底面可看作是刚 性地基, 所以可不设置静态边界, 仅在模型四周布设 自由场边界, 模拟无限场地效果, 边界条件设置情况 如图4所示.

为保证模型边界设置的合理性,在正式计算之前 进行了动力试算, 试算结果表明: 自由场网格与其相 邻的边坡主体网格位移基本连续; 模型底部布设的监 测点所得到的速度时程与计算输入的速度时程曲线 完全吻合, 模型的动力边界设置是合理的.

\section{4 动力参数的输入}

地震波选取 $x$ 方向的简谐波, 波形如图 5 , 速度时程 的峰值为 $0.05 \mathrm{~m} / \mathrm{s}$, 加速度峰值为 $0.15 \mathrm{~g}$, 地震频率 $4 \mathrm{~Hz}$, 从模型底部输入. 阻尼选取局部阻尼, 阻尼系数大小 为 0.156 .

考虑到在简谐地震波作用下针固边坡运动的周 期性特征, 为保证每次提取剪应力时, 边坡的运动状 态相近. 在计算时, 从输入简谐波开始, 每隔一次简谐 波循环提取一次监测点上的剪应力值, 将循环 $n$ 结束 时提取到的监测结果标记为循环 $n$.

\section{5 监测点的选取}

监测点沿界面周向布设如图6所示, 本次数值模

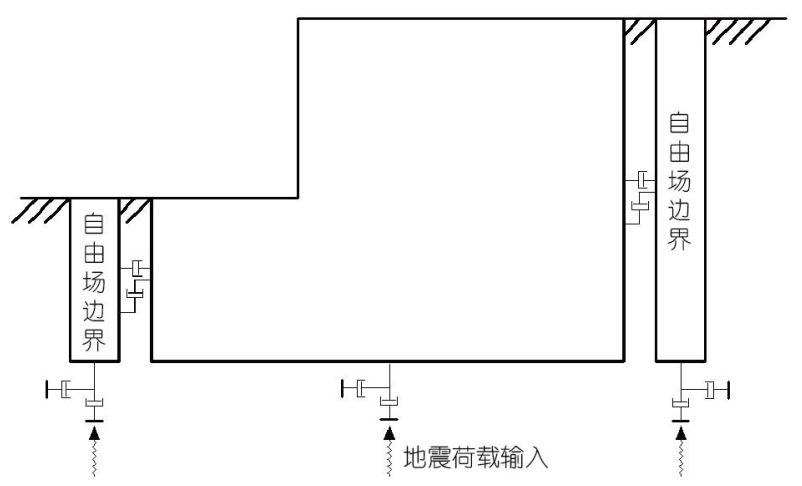

图 4 针固边坡动力边界条件设置图 


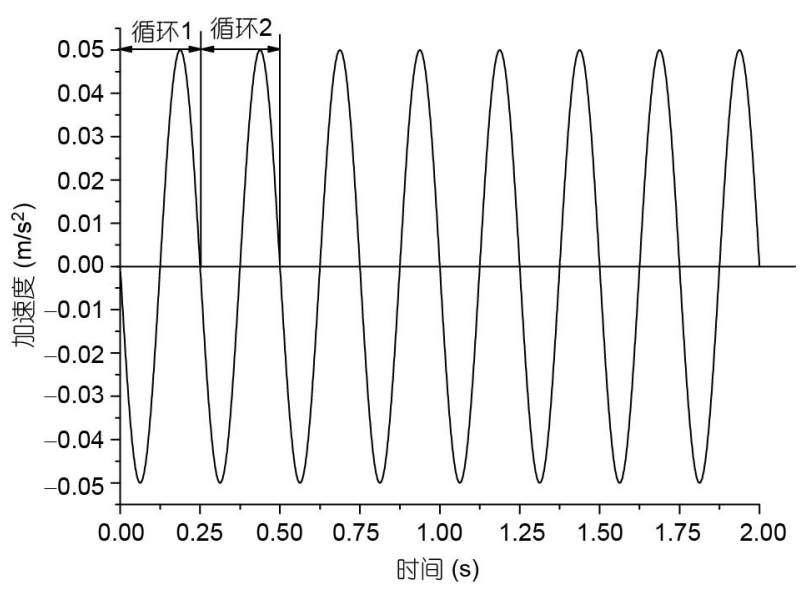

图 5 简谐地震波时程曲线

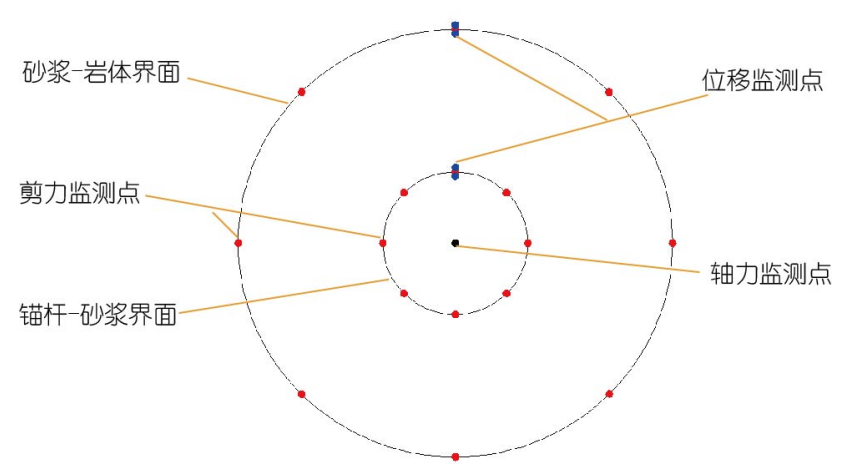

图 6 (网络版彩图)界面周向监测点布设

拟直接提取接触面上的剪应力作为界面剪应力, 经过 试算, 最后沿针杆周向布设 8 个点, 沿针杆轴向每间隔 $0.5 \mathrm{~m}$ 布 1 个点. 可以得到沿针杆轴向和周向的界面剪 应力分布规律.

针杆轴向应力直接提取针杆实体单元中心位置 的轴向应力值, 并换算成轴力.

在研究针杆静力拉拔问题时一般只考虑针杆本 身位移, 本文中为了更好地分析针杆、砂浆、岩体三 者在动力作用下的往复运动对两个界面的剪切相互 作用的影响, 需要同时考虑这三部分的位移. 所以提 取两个界面两侧针杆、砂浆、岩体实体单元节点上 沿针杆轴向的位移.

\section{4 数值模拟结果分析}

\section{1 针固界面剪应力随时间变化规律}

图7为地震作用下针固界面上某一点的剪应力随

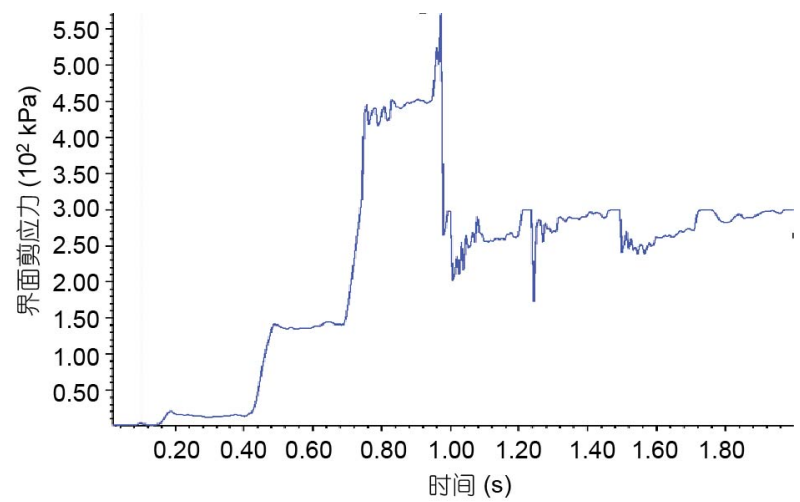

图 7 (网络版彩图)针固界面剪应力随动力加载时间变化 规律曲线

动力加载时间的变化曲线, 该曲线完整地反映了该点 发生剪切破坏的过程: 随着简谐地震荷载的输入, 该 点界面剪应力呈现阶梯式增长, 界面产生弹性变形, 当 该点的界面剪应力达到极限黏结强度后, 界面发生脱 黏破坏, 界面剪应力下降至摩擦阻力. 从曲线波动规 律可以看出, 在地震荷载影响下, 针固界面脱黏前后 监测到的剪应力值都处于波动状态, 而每次循环结束 时提取到的剪应力值也存在波动的可能, 所以为了减 小误差, 在研究界面剪应力沿轴向的分布规律时, 取 针杆某一横截面周向 8 个点的平均剪应力值作为轴向 某位置在该时刻的界面剪应力更加合理.

通过计算发现, 随着地震波的输入, 三根针杆发 生变形破坏的时间有所不同, 但是界面剪应力的分布 和传递规律相似, 因此以布设在边坡顶部的 3 号针杆 为例, 来分析地震作用下针固边坡中针固体针杆-砂浆 界面和砂浆-岩体界面上平均界面剪应力及针杆轴力 的分布规律.

\section{2 岩体和针固体位移规律}

经过计算发现, 针杆与砂浆之间位移的最大差值 不到 $1 \%$, 在分析位移时可以将其看作一个整体, 也说 明针杆和砂浆一直保持同步运动, 针杆- 砂浆界面没 有出现脱黏.

图8(a)中的岩体位移曲线反映了针固区域岩体位 移规律, 随着循环次数的增长, 以 $3.5 \sim 4 \mathrm{~m}$ 位置的软弱 结构面为界, 左侧岩体位移增长速率大于右侧的边坡 基岩, 但是两侧岩体内部位移均保持一致, 说明在针 固区域内岩体仅沿软弱结构面出现破坏, 破坏面左侧 

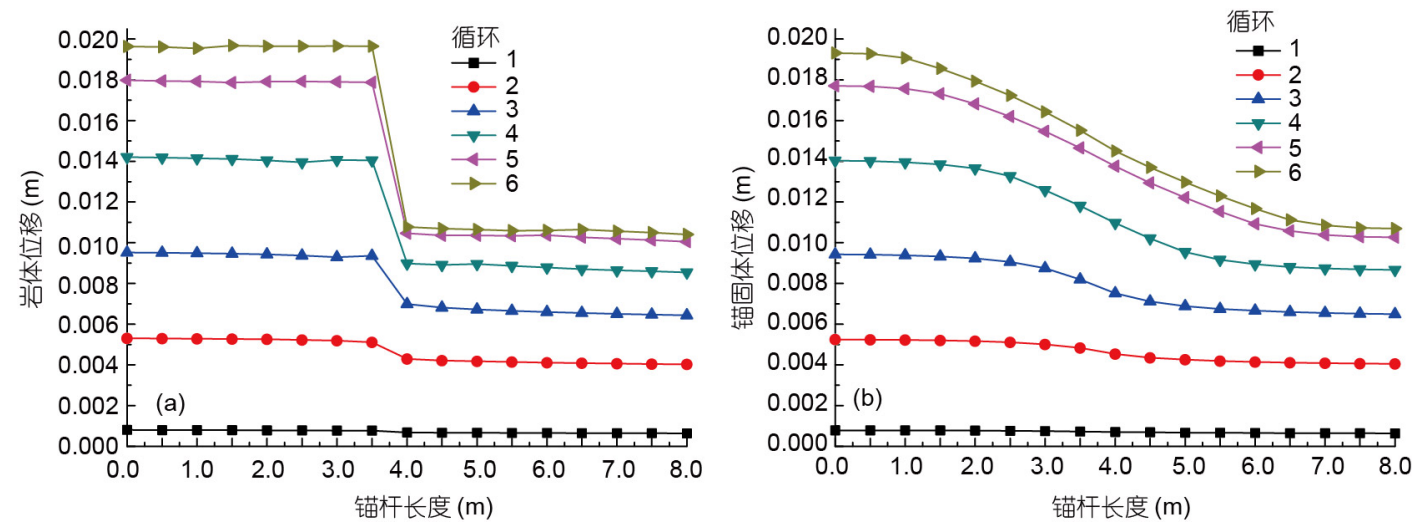

图 8 (网络版彩图)三号针杆周围岩体(a)和针固体(b)的位移曲线

的边坡岩体相对右侧的边坡基岩向外滑移, 成为不稳 定岩体.

将图8(a)岩体位移曲线与图8(b)中的针固体位移 曲线对比可以发现. 在针杆两端, 针固体和岩体的位移 基本一致, 说明该位置的砂浆-岩体界面没有发生脱黏, 该区域为针固体的“固定段”. 在针固体中段, 图8(a)和 (b)对应位移曲线的变化规律不同, 图8(a)岩体位移曲 线为突变式下降, 而对应的图8(b)针固体位移曲线呈 现平滑式下降, 说明中段砂浆-岩体界面出现脱黏, 针 固体与岩体发生较大的相互错动, 错固体受两侧岩体 拉力作用产生均匀拉伸, 该区域为针固体的“拉伸段”. 随着动力加载循环次数的增长, 拉伸段范围逐渐增大.

\section{3 砂浆-岩体界面剪应力分布规律}

如图9所示, 在循环1结束时, 砂浆-岩体界面上的 剪应力峰值很小, 且分布较为均匀, 说明此时砂浆和 岩体之间没有出现明显的相对位移, 针固系统处于稳 定状态。

当循环 2 结束时, 边坡中的软弱结构面已经出现 破坏, 变为破坏面. 不稳定岩体通过针固体将力传递 到边坡基岩中, 两部分岩体中的针固体分别受到了一 个对面针固体施加的, 朝向破坏面的拉力作用, 锚固 体通过杆体本身、针杆-砂浆界面黏结强度和砂浆-岩 体界面黏结强度三者共同作用来抵抗施加在针杆上 的拉力, 从而开始发挥针固作用. 此时砂浆-岩体界面 剪应力在破坏面两侧出现了两个明显的峰值, 两个峰 值剪应力大小相近, 方向相反.

在循环3结束后, 砂浆-岩体界面上的剪应力不断 增大, 其中破坏面右侧砂浆-岩体界面剪应力接近该界

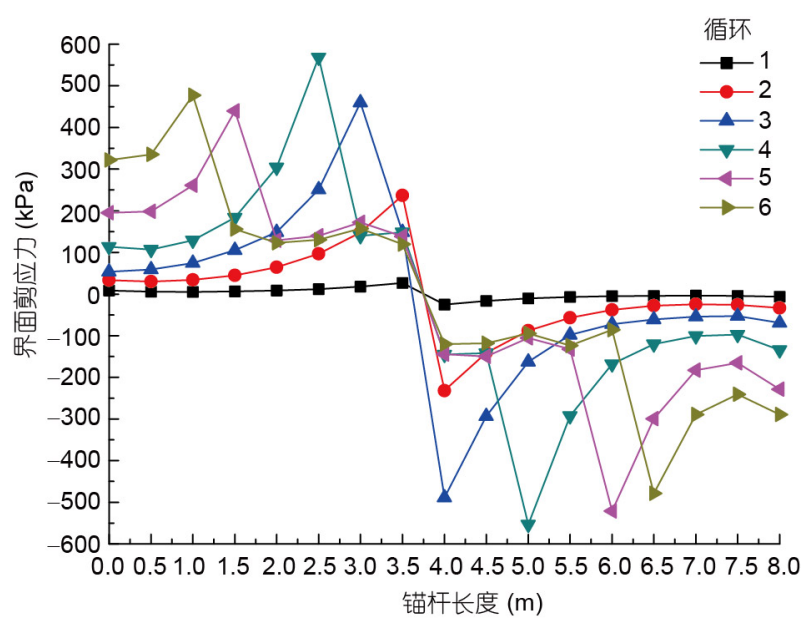

图 9 (网络版彩图)三号针杆砂浆-岩体界面剪应力分布曲 线

面的极限黏结强度, 而破坏面左侧砂浆-岩体界面剪应 力已经超过了该界面的极限黏结强度, 此时破坏面左 侧, 位于针杆 $3.5 \mathrm{~m}$ 处的砂浆-岩体界面出现脱黏现象, 剪应力下降, 剪应力峰值开始向针头位置(孔口)移动.

随着简谐地震荷载的持续输入, 边坡简谐振动次 数的增加, 从循环 4 到循环 6 , 砂浆-岩体界面从破坏面 向针杆两端逐步发生脱黏, 砂浆-岩体界面上左右两 侧的剪应力峰值都从紧挨破坏面的位置向远离破坏 面的方向移动, 孔口和孔底处的剪应力快速增大. 直 到针杆其中一端砂浆-岩体界面全部脱黏, 此时脱黏 的锚固体与岩体之间的界面上仅剩摩擦阻力作用, 锚 固系统失效.

\section{4 针杆-砂浆界面剪应力分布规律}

对比图9和10中循环1结束时两个界面剪应力可 


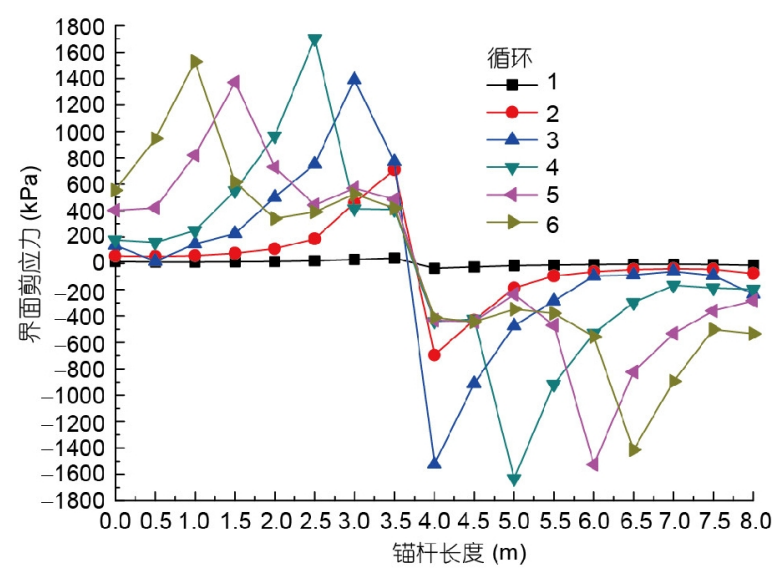

图 10 (网络版彩图)三号针杆杆体-砂浆界面剪应力分布 曲线

以发现, 此时砂浆-岩体界面上的剪应力与针杆-砂浆 界面上的剪应力在数值上大小相近, 说明在施加简谐 地震波的初始阶段, 边坡中还未出现明显破坏面, 两 个界面仅随着针杆、砂浆、岩体三者的共同运动而 产生协调变形, 并没有相互错动, 所以该阶段两个界 面上的剪应力分布比较均匀, 与静力作用下的剪应力 相差甚微.

循环 2 结束时, 针杆-砂浆界面剪应力在软弱结构 面两侧出现了两个峰值, 两个峰值剪应力大小相近, 方向相反. 此时针杆-砂浆界面剪应力峰值在数值上 已经超过了砂浆-岩体界面, 但没有到达针杆-砂浆界 面的极限黏结强度, 所以剪应力继续增长.

循环3结束时, 砂浆-岩体界面从破坏面位置开始 向针杆两端出现脱黏现象, 而此时针杆-砂浆界面上的 剪应力峰值仅为 $1.5 \mathrm{MPa}$, 没有达到该界面的极限黏结 强度2.4 MPa, 因此针杆-砂浆界面依旧处于弹性状态, 并没有出现脱黏现象. 但是针杆-砂浆界面剪应力峰 值也随着砂浆-岩体界面的脱黏而出现相应的调整转 移, 峰值点从破坏面位置向针杆两端移动.

\section{5 针杆轴力分布规律}

图11为三号针杆的轴力分布曲线图, 从图中可以 注意到, 针杆的轴力沿针杆轴向呈人字形曲线分布, 轴力峰值一直在针杆中段软弱结构面位置, 此处的针 杆杆体受到来自两侧砂浆、岩体拉力的联合作用. 从 循环 1 到循环 3 , 两侧针固界面上剪切作用加剧, 锚杆 轴力峰值的增长速度逐渐上升.

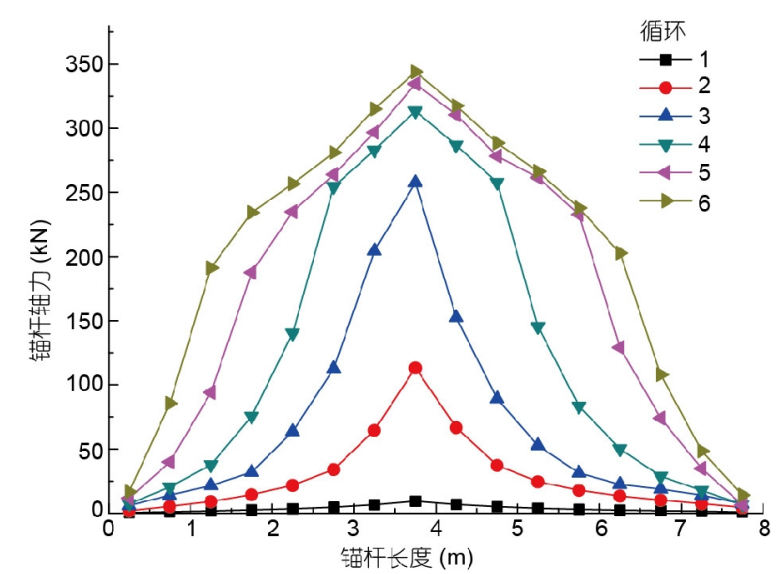

图 11 (网络版彩图)三号针杆轴力分布曲线

随着简谐地震荷载的持续输入, 从循环 4 到循环 6 , 砂浆-岩体界面逐渐脱黏, 而针杆轴力人字形曲线的 两翼也从内凹曲线转变为外凸曲线. 外凸人字形曲线 在凸出部有明显拐点, 以拐点为界, 曲线上半部分轴 力沿轴向变化速度较为缓慢, 峰值基本不再增加, 而 曲线下半部分轴力快速下降.

对比图8中的位移曲线, 图9和10中的界面剪应力 曲线, 并结合图11中的轴力曲线进行分析可以发现, 每 次循环结束时位移曲线的“拉伸段”边界、轴力曲线的 拐点和界面剪应力曲线的峰值点都能够较好地吻合. 说明随着砂浆-岩体界面的脱黏, 原本由两个界面和杆 体共同承担的荷载, 变为仅由针杆杆体和针杆-砂浆界 面承担, 因此在新脱黏区域轴力快速增长, 造成该段 针固体发生均匀拉伸, 针杆轴力在界面脱黏区域的分 布趋于均匀.

根据以上研究可知, 区别于针杆受到静力拉拔时 轴力呈现指数型曲线分布, 界面剪应力呈现单峰值曲 线的分布规律, 地震作用下针固边坡中针杆的轴力呈 现出两翼从内凹到外凸的人字形曲线分布, 而两个界 面剪应力则呈现出双峰曲线的分布形态. 因此在进行 动力针固边坡界面剪切问题试验研究的设计时, 应在 针杆靠近潜在滑动面的位置上布设较多的测试元件, 以得到更加精确可靠的结果. 动力条件下的针杆界面 剪应力问题远比静力拉拔的条件下复杂, 未来还需要 针对不同的情况进行更多研究.

\section{5 结论}

本文通过FLAC ${ }^{3 \mathrm{D}}$ 数值模拟软件建立锚固垂直边 
坡实体单元-接触面单元模型, 将其运用于简谐地震波 作用下针固岩体边坡中两针固界面剪应力的分析研 究, 同时获得了动力作用下针固边坡中锚杆-砂浆、砂 浆-岩体两个界面上剪应力的分布规律, 揭示了两个界 面上的相互作用机理, 为针固边坡相关的模型试验、 现场监测、设计施工提供了重要参考. 结论如下.

(1) 通过该方法建立的针固垂直边坡模型, 锚固界 面剪应力到达极限黏结强度后, 出现脱黏现象, 界面 剪应力下降至摩擦阻力.

(2) 两个界面中砂浆-岩体界面剪应力首先达到该 界面的极限黏结强度并发生脱黏, 界面剪应力的峰值
从破坏面向针杆两端发展.

(3) 当砂浆-岩体界面发生破坏时, 针杆-砂浆界面 上的剪应力并没有达到该界面的极限黏结强度, 界面 本身并没有发生脱黏现象, 但是该界面剪应力峰值也 随着砂浆-岩体界面的脱黏而发生相应的调整转移.

(4) 针杆轴力呈人字形分布, 随着砂浆-岩体界面 的脱黏, 轴力人字形曲线的两翼从内凹曲线转变为外 凸曲线, 轴力曲线拐点与界面剪应力曲线峰值点相对 应, 界面脱黏区域的针固体与岩体之间出现较大的相 对位移, 针固体发生均匀拉伸, 脱黏区域的针杆轴力 分布趋于均匀.

\section{参考文献}

1 程良奎, 范景伦, 韩军, 等. 岩土针固. 北京: 中国建筑工业出版社, 2003. 8-11

2 尤春安. 全长黏结式针杆的受力分析. 岩石力学与工程学报, 2000, 19: 339-341

3 雒亿平, 史盛, 言志信. 抗拔荷载作用下针固体与岩土体界面剪切作用. 煤炭学报, 2015, 40: 58-64

4 任非凡, 徐超, 谌文武. 多界面复合针杆荷载传递机制的数值模拟. 同济大学学报(自然科学版), 2011, 39: 1753-1759

5 黄明华, 周智, 欧进萍. 全长黏结式针杆针固段荷载传递机制非线性分析. 岩石力学与工程学报, 2014, 33: 3992-3997

6 叶海林, 郑颖人, 黄润秋, 等. 针杆支护岩质边坡地震动力响应分析. 后勤工程学院学报, 2010, 26: 1-7

7 Zou X K, Zhang J C, Shi H C, et al. Effect analysis of soft rock slope reinforced by bolts under blasting dynamic load. In: IACGE 2013: Challenges and Recent Advances in Geotechnical and Seismic Research and Practices. Chengdu, 2013. 438-446

8 Mortazavi A, Tabatabaei Alavi F. A numerical study of the behavior of fully grouted rockbolts under dynamic loading. Soil Dyn Earthq Eng, 2013, 54: 66-72

9 Yan Z X, Duan J, Jiang P, et al. Finite difference method for dynamic response analysis of anchorage system. J Cent South Univ, 2014, 21: 1098-1106

10 刘建华, 汪优, 付康林, 等. 简谐振动荷载下针杆加固岩质边坡的受力分析. 岩土力学, 2012, 33: 85-90

11 言志信, 张刘平, 江平, 等. 针固上覆红黏土岩体边坡的地震动力响应. 岩土力学, 2014, 35: 753-758

12 Shen D, Ji Y, Yin F, et al. Dynamic bond stress-slip relationship between basalt FRP sheet and concrete under initial static loading. J Compos Constr, 2015, 19: 04015012

13 韩军, 陈强, 刘元坤, 等. 针杆灌浆体与岩(土)体间的黏结强度. 岩石力学与工程学报, 2005, 24: 3482-3486

14 中华人民共和国建设部. 建筑边坡工程技术规范GB50330-2013. 北京: 中国建筑工业出版社, 2013

15 谈一评, 曾镇强. 简谐动力波作用下错杆拉拔试验研究. 岩土工程学报, 2013, 35: 409-414

16 张建经, 廖蔚芳, 欧阳芳, 等. 重复荷载作用下岩针体系力学特性和黍结性能试验研究. 岩石力学与工程学报, 2013, 32: 829-834

17 Itasca Consulting Group, Inc. FLAC (Fast Lagrangian Analysis of Continua 3D) user's manual. Version 5.0. Minneapolis: Itasca Consulting Group, Inc., 2012 


\title{
A numeral simulation of anchored interface shear stress for bolt in rock mass slope under earthquake
}

\author{
LONG Zhe ${ }^{1}$, YAN ZhiXin ${ }^{1,2} \&$ ZHOU XiaoLiang ${ }^{1}$ \\ ${ }^{1}$ School of Civil Engineering and Mechanics, Lanzhou University, Lanzhou, 730000, China; \\ ${ }^{2}$ School of Civil and Traffic Engineering, Henan University of Urban Construction, Pingdingshan 467000, China
}

In order to extract the distribution regularities of shear stress on both slope's anchored interfaces under earthquake at the same time, and reveal the mechanism of the shear interaction on anchored interfaces. Based on finite difference software FLAC ${ }^{3 \mathrm{D}}$, we build an anchored rock slope numerical model, simulated shear interaction on cable-grout interface and grout-rock interface by interface element, to study the distribution regularities of wholly grouted anchor bar anchored interface shear stress in anchored slope under simple harmonic vibration of seismic waves. The results of the study include the following: the shear stress on grout-rock interface reaches ultimate bond strength first, and debonding, the peak value of shear stress on grout-rock interface develop from failure surface to bolt end; at the same time, the shear stress on bolt-grout interface doesn't reach ultimate bond strength, but the peak value of shear stress on bolt-grout interface move accordingly with that on grout-rock interface; after grout-rock interface debonding, the distribution of axial force along bolt is herringbone curve with both convex outer side, there is a one-to-one correspondence between curve inflection point of axial force and peak value point of shear stress on interface. The anchorage body in debonding region subjected uniform tension, the axial force in this region distributed relatively uniform. The study can provide references for study of model test, field monitoring, design and construction on relevant aspect.

anchored slope, interface element, interface shear stress, bolt-grout interface, grout-rock interface

doi: $10.1360 /$ N092017-00171 